\title{
Perception versus attention
}

Understanding others and being understood in turn is something that most of us take for granted. Development of this capacity for effective communication requires attention to social stimuli, including speech and body language. People with autism orient poorly to social stimuli, showing a distinct attentional preference for objects over people. This abnormal orientation contributes to the impairment of language that is a hallmark of this increasingly prevalent developmental disorder. As socially meaningful cues are physically complex, it has been hypothesized that subjects with autism might have difficulty encoding and representing sensory information. As reported in Proceedings of the National Academy of Sciences, Ceponiene and colleagues tested this hypothesis in highfunctioning children with autism.

Event-related brain potentials were recorded during presentation of acoustically matched tones and vowels, to compare responses to non-speech and speech sounds, respectively. Presenting these acoustic stimuli as an 'oddball' sequence in which a 'deviant' sound was occasionally substituted for a 'standard' repetitive sound allowed the researchers to discriminate between sensory processing and involuntary attentional orienting.

Surprisingly, test subjects with autism showed no significant abnormalities in sensory processing of either tones or vowels. By contrast, their attentional orienting to deviant sounds was impaired. Interestingly, this impairment was selective for vowel sounds - attentional switching was normal in response to deviant non-speech tones, regardless of their complexity. These results indicate that the impairment of auditory processing that inhibits language development in people with autism lies downstream of sensory processing.

Suzanne Farley

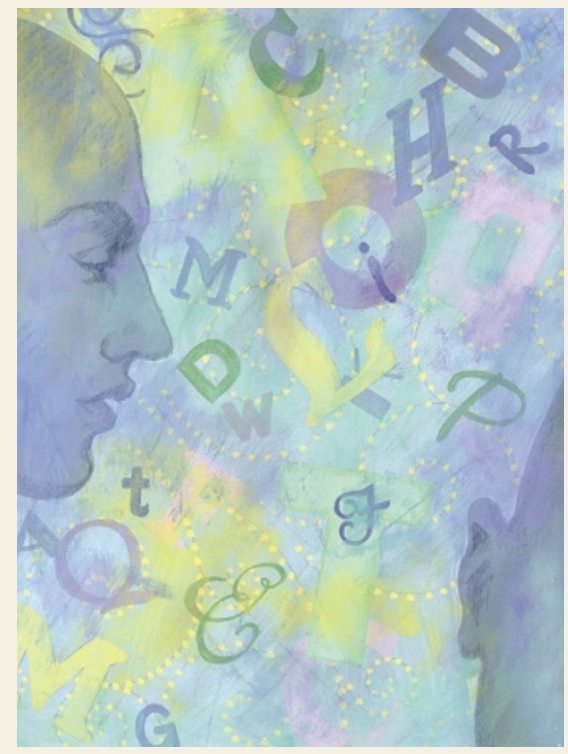

(4) References and links ORIGINAL RESEARCH PAPER Čeponienè, R. et al. Speech-sound-selective auditory impairment in children with autism: they can perceive but do not attend. Proc. Natl Acad. Sci. USA 100, 5567-5572 (2003)

FURTHER READING Siegal, M. \& Varley, R. Neural systems involved in 'theory of mind'. Nature Rev. Neurosci. 3, 463-47 (2002)

\section{DENDRITES}

\section{Stepping stones to synapse formation}

Dendritic spines come in all shapes and sizes, ranging from long and thin, to short and stubby, to mushroom-shaped. Not content with just one form, the regulation of the actin cytoskeleton enables spine morphology to change during the life of a neuron. The molecules involved in regulating actin dynamics are well characterized, but the mechanisms and pathways by which their assembly and localization occur are less clear. In the Journal of Cell

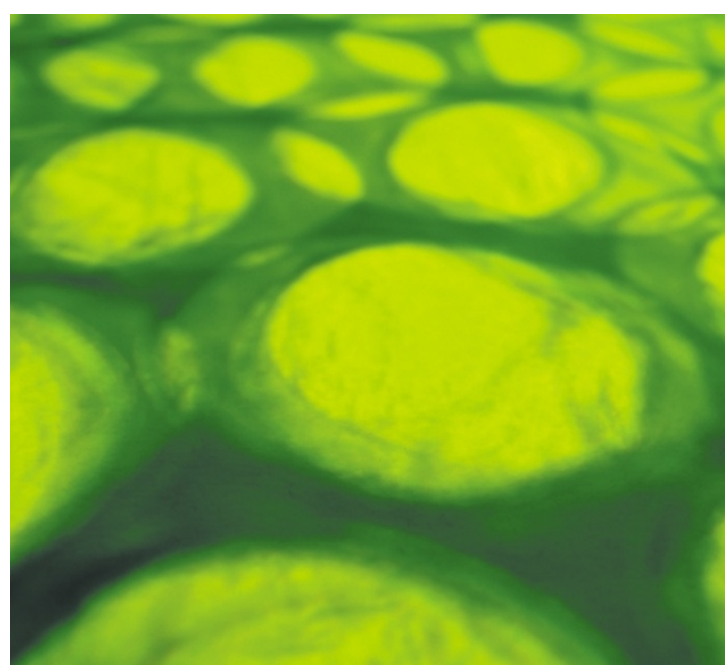

Biology, Zhang et al. describe G-proteincoupled receptor kinase-interacting protein 1 (GIT1), and report on its function as an adaptor protein in the pathway to synapse formation and allocation of spine morphology.

The authors determined that GIT1 localizes to synapses through a synaptic localization domain (SLD), overexpression of which led to a decrease in spine and synapse number, and an increase in the number of long, thin dendritic protrusions (which lacked synapses).

GIT1 can also bind the guanine exchange factor (GEF) PIX. Using a mutant in which the PIX-binding domain had been deleted, the authors found a decrease in spine and synapse formation, a phenotype similar to that seen in neurons overexpressing the SLD. To determine whether GIT1 was responsible for targeting PIX to the synapse, they overexpressed a PIX mutant deficient in GIT1 binding in neurons. The results showed a similar phenotype to that of the PIX-binding mutant - the number of dendritic protrusions (with no associated synapses) increased, and the density of spines and synapses decreased.

PIX is responsible for the activation and regulation of Rac, a member of the Rho family of small GTPases. Is the GEF activity of PIX crucial for correct spine morphology and synapse formation? Zhang et al. found that when GEF activity in PIX was abolished, the number of dendritic protrusions, spines and synapses decreased significantly.

Previously, Nakayama et al. showed that Rac is required for the maintenance of dendritic spines. To examine the role of Rac further, Zhang et al. transfected neurons with a constitutively activated or a dominant-negative form of Rac. The resulting phenotypes showed a significant decrease in synaptic density.

So what is the link between all these different molecules and the dendritic phenotype, and in what order do they participate in the pathway to synapse formation? The authors suggest that the phenotype that results from these mutants is caused by mislocalization of Rac. When GIT1 is mislocalized, it leads to the concurrent mislocalization of activated Rac.

The authors propose that a critical level of localized Rac activation is required for spine and synapse formation and maintenance. When active Rac is not localized, dendritic protrusions persist, and in the absence of active Rac, the protrusions are absent.

Emma Green

(2) References and links

ORIGINAL RESEARCH PAPER Zhang, H. et al. Synapse

formation is regulated by the signaling adapter GIT1. J. Cell Biol. 161, 131-142 (2003)

FURTHER READING Nakayama, A. Y. et al. Small GTPases

Rac and Rho in the maintenance of dendritic spines and branches in hippocampal pyramidal neurons. J. Neurosci. 20 5329-5338 (2000) 